\title{
INVESTIGACIÓN EN COMUNICACIÓN MUNICIPAL: ESTUDIOS Y APORTACIONES ACADÉMICAS
}

\author{
Conchi Campillo-Alhama ${ }^{1}$ : Universidad de Alicante. España
} concepcion.campillo@ua.es

\section{RESUMEN}

Los Ayuntamientos se dirigen a la ciudadanía a través de procesos comunicativos y relacionales para transmitir todo tipo de mensajes sobre la gestión pública; tales procesos se justifican desde la premisa fundamental de que el ciudadano debe estar informado de todas aquellas actuaciones e iniciativas administrativas que pueden incidir en su condición de administrado. La comunicación pública municipal constituye, en este sentido, una poderosa herramienta en manos de los equipos de gobierno que permite explicar, justificar $y$, en consecuencia, legitimar las decisiones políticas asumidas en cada periodo legislativo. En este trabajo se realiza una revisión de las principales aportaciones y contribuciones académicas sobre la comunicación de proximidad, ya que creemos que la investigación académica sobre los procesos de comunicación pública desarrollados en este ámbito puede contribuir, de forma notable, a aumentar la profesionalización de los gabinetes de comunicación en las administraciones locales. Con esta contribución pretendemos, además, facilitar la labor de otros investigadores en el desarrollo de futuros trabajos y estudios vinculados a la comunicación municipal.

PALABRAS CLAVE: Investigación - Comunicación - Municipal - Ayuntamiento Relacional

\footnotetext{
${ }^{1}$ Autor correspondiente

Conchi Campillo-Alhama: Profesora colaboradora Dpto. Comunicación y Psicología Social. Universidad de Alicante, España

Correo: concepcion.campillo@ua.es
} 


\title{
MUNICIPAL COMMUNICATION RESEARCH: ACADEMIC STUDIES AND CONTRIBUTIONS
}

\begin{abstract}
The city councils are directed to the public through communication and relational processes for all types of messages transmitted over public policy, such processes are justified from the fundamental premise that citizens should be informed of all administrative actions and initiatives that may affect in his capacity as given. The municipality public communication is, in this sense, a powerful tool in the hands of the government staff who can explain, justify and, therefore, legitimize political decisions taken in each legislative period. In this paper we review the main contributions and academic contributions on the local communication because we believe that academic research on public communication processes developed in this area can contribute significantly, to increase the professionalism of communication offices in local government. With this contribution we intend to further facilitate the work of other researchers in the development of future work and studies related to municipality communications.
\end{abstract}

KEY WORDS: Research - Communication - Municipality - Town Council - Relational

\section{INTRODUCCIÓN}

En la Administración local, cualquier equipo de gobierno es consciente de que para rentabilizar la gestión política efectuada durante su mandato legislativo es necesario establecer cauces de comunicación a través de los cuáles sus actuaciones se trasladen a la ciudadanía en clave positiva.

El interés que despiertan las organizaciones municipales entre los ciudadanos, tanto por la proximidad de su gestión como por la incidencia de las decisiones políticas en la vida pública local, es mucho mayor que el que se manifiesta respecto a otras instituciones de gobierno. Los Ayuntamientos constituyen así el eje básico de referencia cuando nos aproximamos a la gestión de la vida local. Se dirigen a la ciudadanía a través de procesos comunicativos y relacionales para transmitir todo tipo de mensajes sobre la gestión pública; tales procesos se justifican desde la premisa fundamental de que el ciudadano debe estar informado de todas aquellas actuaciones e iniciativas administrativas que pueden incidir en su condición de administrado. $\mathrm{Y}$, sin duda, la comunicación pública municipal constituye, en este sentido, una poderosa herramienta en manos de los equipos de gobierno que permite explicar, justificar y, en consecuencia, legitimar las decisiones políticas asumidas en cada periodo legislativo.

En esta propuesta presentamos una revisión de la literatura académica existente sobre los procesos de comunicación orientados a los administrados que desarrollan habitualmente los ayuntamientos, incidiendo especialmente en las escasas tesis 
doctorales que han abordado dicho fenómeno y que han contribuido sustancialmente a avanzar en su estudio en España. Nuestro trabajo es una revisión exhaustiva de aportaciones académicas con el objetivo fundamental de facilitar la labor de otros investigadores en futuros proyectos y estudios vinculados a la comunicación de proximidad, que contribuyan, a su vez, a una mayor profesionalización de los gabinetes de comunicación locales.

\subsection{El enfoque contemporáneo de la Teoría de la Organización en la Administración municipal}

Durante la etapa denominada Nueva Gestión Pública (enmarcada a partir de 1999 y hasta la actualidad ${ }^{2}$ de manifiestan dos orientaciones teóricas relacionadas básicamente con la cultura de las organizaciones públicas (Ramió, 1999a, 1999c; Olías de Lima, 2001) y que representan dos formas divergentes de interpretar la gestión político-administrativa: por un lado, nos encontramos con las corrientes neoempresariales, basadas en la economía, la eficacia y eficiencia de los aparatos públicos, que proponen una clientelización de los ciudadanos; y por otro, con las corrientes neopúblicas, las cuáles depositan un especial énfasis en cuestiones como la repolitización de los cargos públicos, la racionalización y control de la externalización de los servicios, la participación ciudadana y la ética en la gestión pública.

El peso específico de estas dos orientaciones es bastante desigual (Ramió, 1999a; Olías de Lima, 2001); así, las corrientes neoempresariales representan la mayoría de la literatura organizativa pública y son la fuente de inspiración directa de muchos equipos de gobierno, especialmente en municipios de gran tamaño poblacional. Las neopúblicas, sin embargo, son corrientes minoritarias tanto en el mundo académico como en las propias bases conceptuales sobre las que se desarrollan la innovación y modernización de la Administración pública.

Ambas se erigen, dentro de la teoría organizativa pública, como las tendencias más significativas que sustentan en la actualidad la política de gestión de los Ayuntamientos; sintetizan los numerosos postulados teóricos que se aplican en el ejercicio de la función pública, muy presentes en el contexto municipal.

La cultura organizativa en el ámbito municipal parte de dos supuestos diferentes (Ramió, 1999c): en un primer supuesto, nos situamos ante un ámbito de gestión en el que existe una ausencia total o bastante acusada de cultura organizativa, y en el cuál se introducen valores neoempresariales; nos encontramos, en este caso, con una ausencia de enfrentamiento entre orientaciones neopúblicas y empresariales,

\footnotetext{
2 Las corporaciones locales democráticas han estado sumergidas en diferentes etapas históricas que arrancan con el modelo burocrático-taylorista, hasta llegar a la perspectiva contemporánea de la nueva gestión pública (NGP) en la que se desarrollan modelos de gestión postburocráticos (Ramió, 1999a, 1999b, 1999c, 2002; Olías de Lima, 2001). Según autores como López Camps (1998), Olías de Lima (2001) y Asensio (2006), podemos distinguir los siguientes periodos: etapa de reorganización (1979-1987), etapa de fomento económico (1987-1991), etapa de eficiencia (1991-1995), etapa de gestión de la calidad (1995-1999) y etapa de la nueva gestión pública (1999 y hasta la actualidad).
} 
emergiendo una cultura organizativa de carácter claramente empresarial. En este contexto, los políticos actuarían bajo un rol semejante a los empresarios, los responsables administrativos como directivos o gerentes y el personal de base asumiría el sistema organizativo para obtener, simplemente, un determinado beneficio económico por su rendimiento laboral. Estamos ante una organización que se concibe como una empresa, en la cual se miden los resultados en términos de eficacia y eficiencia, con la ausencia de la perspectiva organizacional neopública que representa valores como la equidad, la igualdad, la participación ciudadana o los impactos sociales de las políticas desarrolladas.

Existe un segundo supuesto que se refiere al hecho de que cuando la cultura neoempresarial se introduce en organizaciones o ámbitos de gestión pública con una sólida cultura organizativa basada en valores públicos se origina un choque (o al menos cierto enfrentamiento) entre ambas culturas, puede dar como resultado una cultura organizativa híbrida, resultante de la combinación de valores públicos y empresariales. En esta conexión de valores prevalecerán, mayormente, las orientaciones neopúblicas; no obstante, el impulso que las neoempresariales pueden ejercer sobre la gestión de las organizaciones públicas puede ser sumamente positivo, contrarrestando así la defensa de los intereses propios de la organización que van en detrimento de las demandas sociales.

Esta conjunción de orientaciones se traslada a las estructuras organizativas municipales configurando sistemas compuestos por diferentes unidades o subsistemas que se vertebran de manera coordinada en el nivel estratégico para desarrollar funciones de comunicación.

\subsection{La comunicación municipal}

El ámbito local se caracteriza por ser sumamente dinámico; en él intervienen diferentes agentes como los medios de comunicación social, los líderes de opinión, los movimientos asociativos y el tejido social, los ciudadanos individuales y plurales, además de otros niveles administrativos. Los ayuntamientos tienen que adaptarse permanentemente a nuevas circunstancias y exigencias sociales, estableciendo o propiciando procesos de comunicación pública que permitan mostrar (y demostrar) a los ciudadanos que constituyen el epicentro de la actuación administrativa.

En los últimos treinta años, se ha producido en los ayuntamientos una transformación tan radical en su desarrollo competencial y en el ejercicio de la función pública que esta circunstancia ha afectado a las organizaciones locales no sólo a nivel funcional, sino también estructural. En el contexto actual, el antiguo modelo clásicoburocrático, basado en procesos de comunicación unidireccional, ha sido sustituido por modelos de gestión relacional y participativos. La comunicación de los ayuntamientos ya no se asume, exclusivamente, como una comunicación sobre servicios públicos sino desde una perspectiva global de comunicación organizacional a través de la cual se implementa, en el colectivo imaginario, una marca de ciudad con valores propios (Puig, 2003). 
Así, con la llegada de la democracia, emergen, en la estructura orgánica de Fos ayuntamientos, ciertos departamentos (o unidades administrativas) denominados gabinetes de comunicación. A partir de las elecciones de 1979, el nuevo orden político, en el ámbito municipal, exige la creación de servicios comunicativos que posibiliten una conexión permanente con los ciudadanos (Cárdenas, 1999, 2000)33; para ello, se utilizan como agentes intermediarios a los medios de comunicación local (privados o propios).

El hecho de que la Administración municipal se haya convertido en agente de desarrollo económico y garantía del progreso social de una comunidad local, conlleva su intervención en muy diversos ámbitos de la vida cotidiana (Cárdenas, 1999). Está, así, continuamente expuesta a la opinión pública, que puede poner en tela de juicio su imagen, credibilidad y, en consecuencia, su legitimación.

En consecuencia, el hecho de no informar a los ciudadanos o no hacerlo de manera eficaz supone un serio riesgo para la continuidad de los cargos políticos. Tal y como expresa Martínez Bargueño (1985), el objetivo fundamental de los gabinetes de comunicación municipales no es otro que identificar y desarrollar al máximo las relaciones con los ciudadanos, reforzando el conocimiento que éstos poseen de la administración de proximidad para procurar el consenso en torno a la gestión del equipo de gobierno.

A pesar de que las relaciones con los medios de comunicación siguen siendo consideradas como el rol esencial a desarrollar por parte de la dirección de comunicación municipal (Lucas, 1997; Castillo y Almansa, 2001; Almansa, 2004, 2005), se constata cómo dicha función, anclada en los orígenes de los gabinetes, ni es la única ni puede desarrollarse, en el contexto actual, sin el respaldo de otras actividades enfocadas a cubrir las necesidades específicas de la interacción comunicativa-relacional entre administración y administrados ${ }^{4}$

La evolución de los gabinetes de comunicación, en el ámbito de lo municipal, ha venido marcada por una constante adaptación al propio devenir de las estructuras municipales durante toda la etapa democrática. La función comunicacional se ha ido ampliando hasta llegar a configurar estructuras complejas y diversas que presentan, a su vez, profundas dependencias, interrelaciones y sinergias.

\footnotetext{
3 Véase además: Picos Freire (1995), el Reglamento de Organización, Funcionamiento y Régimen Jurídico de las Entidades Locales (1986), la Ley de Bases Reguladora del Régimen Local (1985), la Ley 57/2003, de Medidas para la Modernización del Gobierno Local, y el Anteproyecto de Ley Básica del Gobierno y la Administración Local (2006).

${ }^{4}$ Nos referimos especialmente a la publicidad institucional, la gestión de relaciones institucionales, la participación ciudadana,la atención personalizada a través de las OMACs (oficinas municipales de atención al ciudadano), la comunicación 2.0 y la gestión de acontecimientos especiales, orientados a los ciudadanos como públicos internos, o bien dirigidos a públicos (potenciales) externos, con una notable o trascendente proyección mediática para el municipio
} 
Para planificar y gestionar todas las estrategias y políticas comunicativas del ente municipal, estas unidades o departamentos administrativos se han convertido en auténticas direcciones de comunicación que trabajan con un doble propósito: dar a conocer a los diferentes segmentos de la ciudadanía al ente municipal, como organización que presta servicios y desarrolla actividades de diferente índole; y, por otra parte, propiciar políticas de proximidad y participación ciudadana a través de procesos de comunicación bidireccional: ambos, se plantean con el objetivo final de consolidar una imagen positiva que redunde en la credibilidad y legitimación de la organización pública.

En este sentido, creemos que la investigación académica sobre los procesos de comunicación pública desarrollados en el ámbito municipal puede contribuir, de forma notable, a aumentar la profesionalización de estas unidades administrativas.

\section{METODOLOGÍA}

\subsection{La investigación académica en comunicación municipal}

\subsubsection{Objetivo}

En este trabajo se realiza una revisión de las principales aportaciones y contribuciones académicas sobre la comunicación de proximidad, con el objetivo fundamental de facilitar la orientación de los investigadores que se puedan plantear, en el futuro, proyectos de investigación relacionados con este objeto de estudio.

El estudio que se presenta fue realizado con dos grupos diferentes y en contextos distintos. En el primer caso, se trataba de un grupo de la asignatura de Traducción Científico-Técnica (Universidad Autónoma de Madrid) en el curso 2008-2009. Los estudiantes pertenecían a la Licenciatura de Traducción e Interpretación y cursaban 3ro y 4to. En el segundo caso, se trató de un grupo de 2do curso del Grado de Estudios Ingleses, en la asignatura de Francés III (Universidad de Alcalá).

En el primer caso se trataba de una asignatura en la cual la actividad principal era la traducción y en el segundo el aprendizaje de un idioma, en particular, el Francés. El hecho de tratarse de actividades se ha considerado que aporta elementos positivos en la comparación.

Se pasaron dos cuestionarios, uno en la primera semana y otro en la última. En la mayor parte de los casos los estudiantes tenían la opción de múltiple respuesta así como la posibilidad de formular observaciones.

Entre las preguntas, una relacionada con la propia valoración de las TIC, una vez concluido el curso, es la que presenta quizá una mayor separación en cuanto a los conceptos que interesan en este artículo [Con ellas se aprende más rápido/Con ellas se aprende mejor/Son más entretenidas], y que se puede ver en la tabla adjunta 


\subsubsection{Metodología}

Para ello, recopilaremos los diferentes recursos que hemos hallado sobre comunicación municipal, a partir de las palabras clave: comunicación, ayuntamiento, municipal, comunicación institucional, comunicación pública, marketing público y marketing municipal. A través de un análisis exploratorio realizaremos la búsqueda de diferentes aportaciones académicas relevantes en los repositorios y sitios webs de las universidades españolas en las que se imparten estudios de CC. de la Información/Comunicación, en la base de datos de tesis doctorales Teseo, en las principales revistas académicas del área de comunicación, en portales académicos así como diferentes publicaciones impresas o digitales relacionadas

\section{ANÁLISIS Y DISCUSIÓN}

En este análisis que hemos efectuado sobre la comunicación de los ayuntamientos, hemos hallado diferentes proyectos de investigación y contribuciones académicas que recogemos en las tablas siguientes: en la Tabla 1, se apuntan diferentes memorias de tesis doctoral que centran su ámbito de estudio en la comunicación de proximidad desarrollada por los ayuntamientos españoles y se destacan sus aportaciones más relevantes a modo de síntesis; en la Tabla 2 y 3, se reflejan diferentes contribuciones teóricas sobre el proceso de comunicación pública que desarrollan las administraciones municipales a través de artículos académicos y diversas publicaciones

Tabla 1. Tesis de doctorado

Autor: PÉREZ GONZÁLEZ, R.A. (1988)

Título:

Un modelo estratégico de comunicación municipal. Colección tesis doctorales $n^{\circ}$ 242/88. Universidad Complutense de Madrid

Síntesis:

Este trabajo se centra sobre la estrategia de la comunicación aplicada al ámbito de la ciudad, y para ello el autor establece tres acotaciones: el enfoque estratégico, un análisis comunicacional y el ámbito urbano. El sistema propuesto por Pérez González responde a las necesidades comunicativas y relacionales del Ayuntamiento de Madrid en el año 1982: es la primera propuesta sistematizada en España, relacionada con la gestión de comunicación pública centrada en el contexto más inmediato del ciudadano y responde a las necesidades específicas de una organización municipal instalada en un incipiente contexto democrático. El autor realiza un análisis de la comunicación municipal del Ayuntamiento madrileño aplicando un modelo estratégico basado en seis fases:

a) pública sobre la gestión municipal a través de encuestas.

La investigación de la opinión b)

El análisis de la información aparecida en los medios de comunicación sobre las diferentes áreas de gestión políticoadministrativa. 
c)

La realización de un balance

d) de gestión por parte de la Actministración municipal.

El establecimiento de una estrategia de comunicación del balance de gestión municipal de la legislatura.

e) corporativa y señalización urbana.

El programa de identidad f) estrategia, su supervisión y adaptación.

La implementación de la

Autor: PÉREZ MADRID, J.E. (1990)

Título: Los problemas de comunicación de las administraciones públicas. Tesis Doctoral. Universidad de Navarra

Síntesis:

En ella se plantea que la comunicación de las Administraciones Públicas derivan en una inadecuada articulación de los elementos que forman parte del proceso comunicacional interno: emisor, mensaje y canales. No obstante, el autor considera que los problemas derivados de este ecosistema comunicacional administrativo son fácilmente solventables gracias a la creación y gestión pertinente de redes de comunicación fluidas y sin obstáculos comunicacionales como los sistemas de comunicación y la efectiva comunicación interpersonal.

Pérez Madrid establece que un adecuado ecosistema comunicacional administrativo, formado por redes de comunicación fluidas y estables, la efectiva comunicación interpersonal, así como sistemas de comunicación reales e idóneos, puede ayudar tanto a los administradores (que se sentirán como verdaderos partícipes de un proceso real), como a la gestión de los políticos, superando la presunta e insostenible contradicción entre ambos. En este trabajo se muestran las principales características del proceso comunicacional interno ocasionado por las diferentes tareas y objetivos administrativos, así como la viabilidad de la detección de muchos problemas observados en las organizaciones administrativas públicas.

Autor: RUBIO, L. (2005)

Título: Singularidades de la gestión de la comunicación externa en las organizaciones públicas. Tesis Doctoral. Universitat Ramón Lluch.

\section{Síntesis:}

La aportación fundamental de este trabajo es la propuesta de un modelo diseñado a partir de la sistematización de los procesos de comunicación pública (concretamente, de mensajes de publicidad institucional) que se producen en los Ayuntamientos de Irún, Sant Cugat y El Vendrell.

Se basa en tres registros diferenciados que, según la autora, se producen en los procesos de comunicación pública de los ayuntamientos: el operativo, el de liderazgo y el de socialización e identificación. El primero de ellos, el operativo, se vincula a la prestación de servicios públicos por parte del ente municipal; el segundo, identifica a la institución pública como un sistema global y como agente impulsor de los cambios sociales desde una posición de liderazgo; mientras que, a través del tercer círculo concéntrico, se otorga valor añadido a los conceptos vinculados a los otros dos ámbitos. Las acciones comunicativas que se enmarcan en el registro operativo forman en sí mismas parte de la misma prestación, facilitando su conocimiento, la 
accesibilidad y las relaciones que se establecen con motivo de dicha prestación. Se basa en la información sobre los derechos y obligaciones de los ciudadanos, así como en el funcionamiento y la operativa propia de los servicios municipales. Las actividades desarrolladas desde el registro de liderazgo se asocian a los objetivos de motivación de los agentes implicados, a la innovación y al cambio social como premisas fundamentales, así como al mantenimiento de las redes sociales. Este registro se articula mediante cuatro vectores: la comunicación corporativa que consolida una imagen global de la organización proyectando una personalidad única que permita generar confianza y credibilidad, sintetizando la identidad proyectada hacia el exterior con la identidad de la propia organización; la promoción de cambios en los comportamientos y valores sociales, que identifica a las organizaciones públicas como agentes promotores del cambio de la sociedad desde una posición de

liderazgo social; la visión organizacional o proyecto comunitario que se corresponde con todo aquello que se comparte con la ciudadanía desde el proyecto ideológico del equipo de gobierno; y, el vector relacional que reorienta a las organizaciones municipales a desarrollar su trabajo en asociación con otros agentes sociales, económicos y políticos, configurando redes de colaboración permanentes para crear y compartir valores e intereses comunes a través de la participación ciudadana; este cuarto vector se identifica con cualquier actividad que promueva o facilite los espacios de escucha, diálogo y deliberación necesarios para generar un cierto sentido colectivo del interés público. A través del registro de socialización e identificación las organizaciones municipales estimulan el sentido de pertenencia de los ciudadanos a una determinada comunidad local y favorecen la formación de ciudadanos activos y responsables; este tercer registro se manifiesta mediante vínculos emocionales con lo comunitario, pero además cumple una función de educación civil, ya que fortalece a la ciudadanía facilitando la comprensión de los procesos de gobierno público y favoreciendo la aceptación de intereses colectivos.

Autor: CAVADAS, M.J. (2010)

Título: Análisis de las comunicación municipal en las principales capitales europeas. Tesis Doctoral. Universidad Complutense de Madrid.

\section{Síntesis:}

En esta propuesta se analiza la comunicación como elemento estratégico dentro de la gestión de los gobiernos locales y se constata que la aparición de Internet ha multiplicado la capacidad de influencia de los entes municipales. Según la autora, las webs locales se han convertido en una herramienta muy útil para promocionar el municipio en el plano nacional e internacional y difundir los logros de la gestión política a los ciudadanos. Añade que las características de la red aportan tal complejidad al proceso que esta circunstancia ha terminado por afectar las relaciones de los ciudadanos con los medios, y de éstos con los gobiernos locales. Lo municipal ocupa un espacio propio dentro de la especialización informativa y, así, los gobiernos locales han encontrado en la información sobre su gestión una excelente herramienta de marketing. Este trabajo analiza la interrelación entre los gobernantes locales y los ciudadanos, a través de los portales municipales de Internet. El proyecto se delimita a partir de las capitales de los países europeos con mayor población: Alemania (Berlín), Francia (París ), Reino Unido (Londres ), Italia (Roma ), España (Madrid ), Polonia (Varsovia ), Rumania (Bucarest ), Holanda (Ámsterdam ), Grecia (Atenas ), Portugal (Lisboa ) y Bélgica (Bruselas). 
Tabla 2. Artículos académicos

Autor: ALGUACIL, J. (2005): “Los desafíos del nuevo poder local: la participación como estrategia relacional en el gobierno local", en Polis, Revista Académica de la Universidad Bolivariana, No 12

Autor: CAMPILLO. C. (2010): “Comunicación pública y administración municipal. Una propuesta de modelo estructural", en Pensar la Publicidad, vol. 4, no 1, pp. 45-62.

Autor: CAMPILLO. C. (2011): “La articulación de la gestión política a través de la planificación estratégica de las relaciones informativas. Un estudio exploratorio sobre la agenda temática en el municipio de Elche", en Fisec Estrategias, año VI, n¹5, pp. 125-146.

Autor: CANTALAPIEDRA, M.J. y otros (2007): "Webs municipales del País Vasco y Navarra: importancia de la dimensión de servicios y retraso en el aspecto informativo", en Estudios sobre el mensaje periodístico, $\mathrm{N}^{\mathrm{o}} 13$, pp. 13-30.

Autor: CÁRDENAS, M.L. (2005): "El gabinete de comunicación en la administración electrónica", en Razón y palabra, $\mathrm{N}^{\circ} 45$.

Autor: CERVERA, A. (2001): "La gestión del marketing en la administración pública local", en Revista de Dirección, organización y administración de empresas, No26, pp: 113-124.

Autor: DE MORAGAS, M. y otros (2002): "Comunicación y Ayuntamientos en la era digital", en Lecciones del Portal, Portal de la comunicación, Institut de la Comunicació, UAB.

http://www.portalcomunicacion.com.

Autor: DE SAN EUGENIO, J. (2008): “Comunicación y territorio", en Lecciones del Portal, Portal de la comunicación, Institut de la Comunicació, UAB. http:/ / www.portalcomunicacion.com.

Autor: GÓMEZ RODRÍGUEZ, P.M. (2003): "La gestión de marketing de ciudades y áreas metropolitanas: de la orientación al producto a la orientación al marketing", en Cuadernos de Gestión, vol. 3, no 1 y 2, pp: 11-25.

Autor: LARRANAGA ZUBIZARRETA, J. (2005): “Aproximación a la política de comunicación en los ayuntamientos de la COMUNIDAD Autónoma Vasca”, en Mediatika n 11, pp. 135-150.

Autor: SIXTO GARCÍA, J. (2010): “Marketing de ciudades: las ciudades también se venden, las ciudades también son productos", en Pensar la Publicidad, vol. 4, nº 1, pp. 211-226.

Tabla 3. Publicaciones (Capítulos de libro/ Monografías)

Autor: ADC-DIRCOM (1998): "Comunicarse con el ciudadano", en Los nuevos retos de la comunicación ante el exceso de información: La Comunicación Empresarial e Institucional. Madrid.

Autor: ASENCIO, P. (2008): Marketing municipal. Madrid. Díaz de Santos.

Autor: BEATO, M. (2002): Cauces de comunicación de las administraciones públicas con los ciudadanos. Barcelona. Tecnos.

Autor: BORJA, J. y CASTELL, M. (1997) (7 $7^{\mathrm{a}}$ ed.): Local y global, la gestión de las ciudades en la era de la información. Madrid. Grupo Santillana de ediciones. 
Autor: CANALS, J.M. (1987): "Información para la decisión e información para el ciudadano", en JORDI BORJA Y OTROS, Manual de gestión municipal democrática. Madrid- Barcelona. Instituto de Estudios de Administración Local.

Autor: CÁRDENAS, M.L. (2000): Gabinetes de prensa municipales en la provincia de Sevilla: la información como servicio local. Diputación de Sevilla.

Autor: CARRASCO BELINCHÓN, J. (1977): “La relación administración-administrado en la esfera municipal: análisis de las RR.PP. Municipales", en IV congreso hispano-lusoamericano-filipino de municipios. Barcelona.

Autor: CASTILLO A. y ALMANSA, A. (2001): “El gabinete de comunicación en el ámbito local", en R. LÓPEZ LITA y otros, La prensa local y la prensa gratuita.Castellón. Publicacions de la Universitat Jaime I.

Autor: COSTA BADÍA, P.O. (2001): "Comunicación Pública en el ámbito local", en . BENAVIDES (dtor.), Dirección de comunicación empresarial e institucional. Barcelona. Autor: DE ELIZAGARATE, V. (2003): Marketing de ciudades. Madrid. Pirámide/Esic.

Autor: DE LA CUADRA-SALCEDO, T. (2002) (coord.): "Comunicación y Corporaciones Locales", en El régimen jurídico de la Comunicación Local. Barcelona. Marcial Pons.

Autor: DÍEZ LOBO, J. (2004): "La información de la entidades locales", en J.I. BEL MALLÉN (coord.), Comunicar para crear valor. La dirección de comunicación en las organizaciones. Navarra. Eunsa.

Autor: FERNÁNDEZ RAMOS, S. (2005): La información y participación ciudadana en la Administración local. Barcelona. Bosch.

Autor: FORN I FOXA, M. (1988): Sistemas de información y gestión para la administración Local. Madrid. Instituto Nacional de la Administración Pública (INAP).

Autor: FVMP (Federación Valenciana de Municipios y Provincias) (1995b): Comunicación local en la Comunidad Valenciana, monográfico no 15 . Valencia. Publicaciones FVMP.

Autor: GALÁN, A. (2000): "Comunicación Pública", en J. TORNOS y A. GALÁN (coords.), La Comunicación pública: información administrativa al ciudadano. Madrid. Marcial Pons.

Autor: LÉON VERGARA, M.R. (2006): "Los gabinetes de comunicación en la Administración local: servicio público o servicio partidario", en R. LÓPEZ LITA y otros, La comunicación corporativa en el ámbito local. Castellón. Publicacions de la Universitat Jaime I.

Autor: MAP- MINISTERIO PARA LAS ADMINISTRACIONES PÚBLICAS (2006): "Comunicación y marketing público dirigido al ciudadano", en Guías de apoyo a la calidad en la gestión pública local, $\mathrm{n}^{\mathrm{0}} 10$.

Autor: MORENO, A. y CORCOY M. (2001): "Las corporaciones municipales y la información pública (1979-2001)", en R. LÓPEZ LITA y otros, La prensa local y la prensa gratuita. Castellón. Publicacions de la Universitat Jaime I.

Autor: MOUTERDE, P. (1998): "Comunicarse con el ciudadano", en Los nuevos retos de la comunicación ante el exceso de información: La comunicación empresarial e institucional. Madrid. ADC-Dircom.

Autor: PUIG, T. (2003): La comunicación municipal cómplice con los ciudadanos. Somos una marca de servicios pública con propuestas innovadoras y un estilo entusiasta. Barcelona. Paidós Comunicación. 
Autor: SABÉS, F. y VERÓN, J.J. (2008): La gestión de la información en la administración local. Sevilla, Zamora. Comunicación Social.

Autor: SEISDEDOS, G. (2007): Cómo gestionar las ciudades del S. XXI. Del "city marketing" al "urban management". Madrid. Prentice Hall Financial Times.

Autor: TORNOS, J. Y GALÁN, A. (coords.) (2000): Comunicación pública: la información administrativa al ciudadano. Madrid. Marcial Pons.

Autor: ZURUTUZA, C. (2006): "La comunicación de crisis en ámbitos corporativos locales", en R. LÓPEZ LITA y otros, La comunicación corporativa en el ámbito local. Castellón. Publicacions de la Universitat Jaime I.

\section{CONCLUSIONES}

Tras realizar esta revisión, podemos concluir que la comunicación de proximidad en el contexto académico no ha suscitado en nuestro país un gran interés, dadas las escasas contribuciones y aportaciones teóricas que hemos hallado. Tal es así que durante los últimos veintitrés años (1988-2011), solamente hemos encontrado, tras consultar el repositorio de Tesis doctorales del Ministerio de Educación Teseo, cinco memorias de investigación que se han desarrollado sobre el ámbito de estudio apuntado (Tabla 1): de entre ellas, la de Pérez González (1988), la de Rubio (2005) así como nuestra propia aportación (Campillo, 2009) se configuran a partir de modelos de comunicación municipal para contextos locales específicos, desde la orientación de la estrategia, del proceso comunicativo o de los sistemas, sus estructuras y funciones, respectivamente. Por su parte, la aportación de Pérez Madrid (1990) se delimita de forma exclusiva al ámbito interno de las administraciones públicas y, por extensión, a las dificultades de comunicación interna que se producen en los procesos derivados de esta interacción entre empleados públicos y la clase política; mientras que, en la tesis de Cavadas (2010), nos encontramos una aproximación a la comunicación municipal a través de las webs de los ayuntamientos de las principales capitales europeas.

Creemos, en consecuencia, que existe todavía mucho recorrido en el estudio y el análisis de la comunicación municipal como interacción relacional de los ayuntamientos con sus ciudadanos-administrados. Sin duda, el peso específico de la doctrina académica sobre comunicación pública municipal es mucho menor que el de otros sectores comunicativos, tal y como hemos podido constatar recopilando las escasas publicaciones sectoriales y artículos académicos que aparecen reflejados en la Tabla 2 y 3.

Por tanto, podemos afirmar que, en nuestro país, existe un nicho muy importante en la investigación académica sobre la comunicación pública municipal. El desarrollo de diferentes líneas de investigación futuras serían aportaciones muy enriquecedoras que permitirían tanto avanzar en el análisis de este objeto de estudio, complementando la recopilación que hemos efectuado a través de esta revisión de recursos académicos, como contribuir a una mayor profesionalización de los gabinetes de comunicación en las estructuras municipales. 
Cuando se produce un cambio político en el equipo de gobierno municipal, es habitual que los gabinetes de comunicación se desbaraten y se implanten nuevas estructuras o remodelen las ya existentes, asignando nuevas funciones y recursos humanos a tales unidades administrativas. Las actuaciones de seudo-profesionales o agentes implicados en determinados intereses partidistas dentro de tales departamentos, y especialmente en el ámbito de las relaciones informativas, desafortunadamente, es una práctica habitual de la clase política de nuestro país, que cobra especial visibilidad en periodos post-electorales. Y, en este sentido, los responsables municipales deberían garantizar una mayor profesionalización de este servicio que planifica y diseña procesos de gestión comunicativa y relacional. Esta contraposición manifiesta entre "la politización" de las estructuras comunicativas municipales y "su profesionalización" representa un pulso al derecho a la información de los ciudadanos y, por extensión, a la normalización de la vida democrática local. La investigación académica puede contribuir, en este sentido, a garantizar la ejecución de las funciones y procesos relacionados con la comunicación municipal desde una orientación basada en la eficiencia de tales estructuras administrativas.

\section{REFERENCIAS}

Almansa Martínez, A. (2004). Historia de los gabinetes de comunicación en España. Revista Historia y Comunicación Social, (9): 5-21.

Almansa Martínez, A. (2005). Relaciones públicas y gabinetes de comunicación. Anàlisi, (32): 117-132.

Anteproyecto de Ley Básica del Gobierno y la Administración Local (LBGAL), de 3 de mayo de 2006.

Asencio Romero, P. (2006). El libro de la gestión municipal. Claves de éxito para políticos y directivos locales. Madrid: Díaz de Santos.

Cárdenas Rica, M. ${ }^{\text {a }}$ L. (1999). Profesionalización de los gabinetes de prensa municipales. Revista Latina de Comunicación, (15)

Cárdenas Rica, M. ${ }^{a}$ L. (2000). Gabinetes de prensa municipales en la provincia de Sevilla: la información como servicio local. Sevilla: Diputación de Sevilla.

Castillo Esparcia, A. \& Almansa Martínez, A. (2001). El gabinete de comunicación en el ámbito local. En López, R. \& et al. La prensa local y la prensa gratuita Castellón: Universitat Jaime I. pp. 383-391

Díez Lobo, J. (2004). La información de las entidades locales. En Bel,J. I. (coord.): Comunicar para crear valor. La dirección de comunicación en las organizaciones. Navarra: Eunsa. pp. 259-271

Ley 7/1985, de 2 de abril, Reguladora de las Bases del Régimen Local. 
Ley 57/2003, de 16 de diciembre, de medidas para la modernización del gobierno local.

Lucas Marín, A. (1997). La comunicación en la empresa y en las organizaciones. Barcelona: Bosch Comunicación.

Martínez Bargueño, M.1 (1985). Información administrativa un derecho constitucional. En AA.VV. Información Institucional. Primeros encuentros. Valencia: Generalitat Valenciana.

Olías de Lima, B. (2001) (coord.). La nueva gestión pública. Madrid: Pearson Educación

Picos Freire, J. J. (1995). Estructura de la prensa local en España. En AA.VV. Manual de Periodismo. Barcelona: Prensa Ibérica. pp. 608

Puig Ricart, T. (2003). La comunicación municipal cómplice con los ciudadanos. Somos una marca de servicios pública con propuestas innovadoras y un estilo entusiasta. Barcelona: Paidós Comunicación.

Ramió Matas, C. (1999a). Teoría de la organización y administración pública. Barcelona: Tecnos/Universitat Pompeu Fabra.

Ramió Matas, C. (1999b). Desarrollo organizativo del Ayuntamiento Gerencial.Barcelona: Universitat Pompeu Fabra.

Ramió Matas, C. (1999c). Corrientes neoempresariales versus corrientes neopúblicas: cultura administrativa, valores públicos y credibilidad social. Un planteamiento radical. Instituciones y Desarrollo, (5). Barcelona: Institut Internacional de la Gobernabilitat de Catalunya.

Ramió Matas, C. (2002). E-Administración i nous models de gestió pública. En Barcelona, I Congrès Català de Gestió Pública..

Real Decreto 2568/1986, de 28 de noviembre, Reglamento de organización, funcionamiento y régimen jurídico de las Entidades locales.

\section{Conchi Campillo-Alhama}

Profesora colaboradora del Dpto. de Comunicación y Psicología Social de la Universidad de Alicante. Licenciada en CC. de la Información (Publicidad y Relaciones Públicas) por la U.C.M. Doctora en Sociología por la Universidad de Alicante. Experta en Protocolo y RR. Ha desempeñado su actividad profesional en varias organizaciones públicas y privadas. Pertenece al "Grupo de investigación de estudios de publicidad institucional, política y social" y a "Comunicación y públicos específicos", abordando líneas de investigación como la comunicación pública de proximidad, la gestión de las relaciones informativas, la agenda mediática y temática local, o comunicación y desarrollo local. 